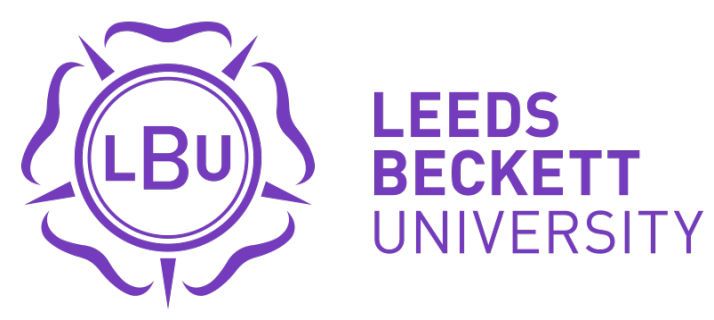

Citation:

Tipples, J (2017) Caution Follows Fear: Evidence from Hierarchical Drift Diffusion Modelling. Emotion. ISSN 1528-3542 DOI: https://doi.org/10.1037/emo0000342

Link to Leeds Beckett Repository record:

https://eprints.leedsbeckett.ac.uk/id/eprint/3814/

Document Version:

Article (Accepted Version)

The aim of the Leeds Beckett Repository is to provide open access to our research, as required by funder policies and permitted by publishers and copyright law.

The Leeds Beckett repository holds a wide range of publications, each of which has been checked for copyright and the relevant embargo period has been applied by the Research Services team.

We operate on a standard take-down policy. If you are the author or publisher of an output and you would like it removed from the repository, please contact us and we will investigate on a case-by-case basis.

Each thesis in the repository has been cleared where necessary by the author for third party copyright. If you would like a thesis to be removed from the repository or believe there is an issue with copyright, please contact us on openaccess@leedsbeckett.ac.uk and we will investigate on a case-by-case basis. 


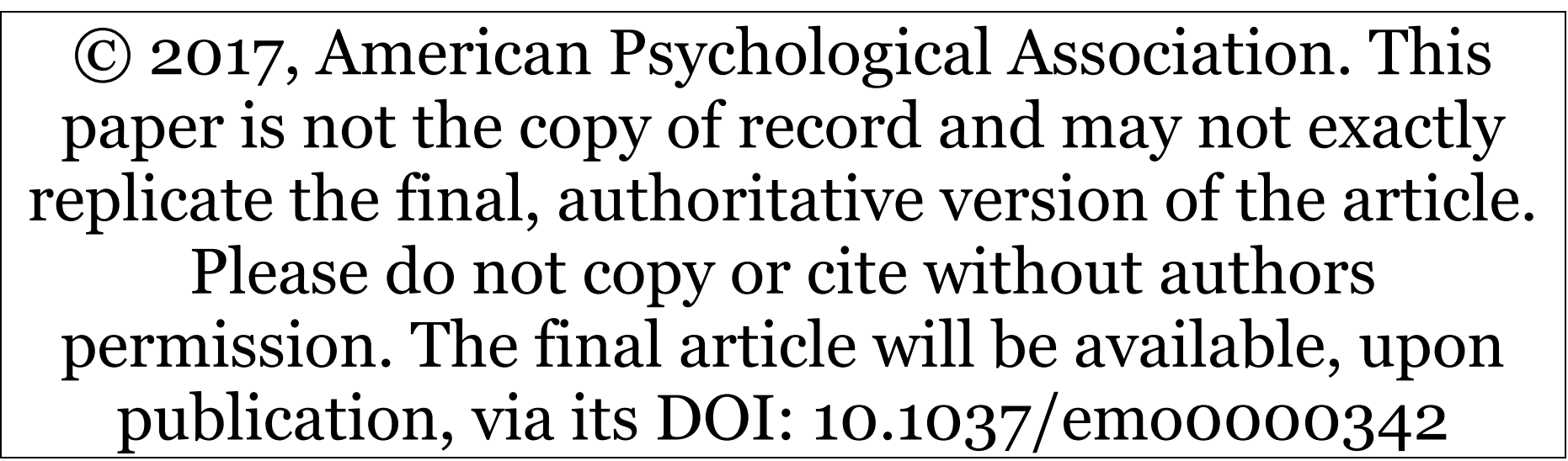

Caution Follows Fear: Evidence from Hierarchical Drift Diffusion Modelling

\author{
Jason Tipples \\ Psychology Group, \\ School of Social, Psychological \& Communication Sciences \\ Leeds Beckett University
}

Requests for reprints should be addressed to: Jason Tipples, Psychology Group, School of Social, Psychological \& Communication Sciences Leeds Beckett University, [CL 815], City Campus, Leeds, LS1 3HE, UK. Tel: +44 (o)113 81223002 | Email: W.Tipples@leedsbeckett.ac.uk

\begin{abstract}
Fearful expressions are thought to warn of potential threat (Davis et al., 2011; Whalen, 1998) and therefore, one response to seeing fear might be to react cautiously. Although previous studies have tested for an effect of seeing fear on visual perception, they have not tested for increased decision making caution. Here, I applied Hierarchical Drift Diffusion Modelling (Vandekerckhove, Tuerlinckx, \& Lee, 2011a; Wiecki, Sofer, \& Frank, 2013) to the results of 4 experiments designed to test the idea that seeing facial expressions both impairs visual perception (Bocanegra \& Zeelenberg, 2009a) and leads to changes in decision making caution. Standard statistical analyses showed that reaction times were slower following fearful compared to neutral expressions. Diffusion Modelling showed that the data were best described by increased caution and not impaired perception. Further experiments showed that: 1) happy expressions did not lead to increased caution (Experiment 3) and 2) people were less cautious after seeing sad compared to neutral expressions (Experiment 4). Overall, the results point to a new direction for research in this area - testing for differences in decision making caution following facial expressions and other emotion cues.

\section{Caution Follows Fear: Evidence from Hierarchical Drift Diffusion Modelling}

"A wealth of information creates a poverty of attention" (p. 41-42; Simon, 1971)

Emotion is thought to help solve the problem of too much information by prioritising information relevant to our goals. Such prioritisation is thought to occur at a very early stage of processing by influencing perception and attention. For example, one set of studies have consistently recorded impaired visual perception following the presentation of fearful compared to neutral expressions (Bocanegra \& Zeelenberg, 2009a; Borst \& Kosslyn, 2010a; Nicol, Perrotta, Caliciuri, \& Wachowiak, 2013a). Evidence for an early prioritisation is primarily based on the separate analyses of reaction times (RTs) and accuracy rates. The separate analyses of RTs and accuracy means that researchers may have overlooked one way in which people react to emotion cues - by reacting cautiously - slowing down in an attempt to be more accurate. Testing for
\end{abstract}


increased caution requires analysing the effect of emotion on the joint relationship between accuracy and RTs. Here, I use Hierarchical Drift Diffusion Modelling (Vandekerckhove et al., 2011a; Wiecki et al., 2013) to test for both impaired perception and increased caution following fearful, happy and sad facial expressions.

\section{Facial Expressions and Visual Perception}

Pioneering studies (Bocanegra \& Zeelenberg, 2009; Phelps et al., 2006) have shown that seeing someone expressing fear has a profound effect on basic visual perception. For example, one study (Bocanegra \& Zeelenberg, 2009) examined the effect of presenting a fearful face on observers' sensitivity to the orientation of a Gabor patch. The authors varied the spatial frequency of the targets to examine whether the previous report of enhanced vision (Phelps et al., 2006) due to fear might be restricted to low spatial frequency information (LSF). The findings extended the results of an earlier study (Phelps et al., 2006) by showing that fearful faces both enhanced orientation sensitivity for the low spatial frequency information (LSF) and impaired orientation sensitivity for high spatial frequency information (HSF). Subsequent studies have replicated both enhanced and impaired visual perception following fearful expressions (Bocanegra, Huijding, \& Zeelenberg, 2012; Bocanegra \& Zeelenberg, 2011; Borst \& Kosslyn, 2010a; Nicol et al., 2013a). Moreover, further research showed that the effects are not restricted to fearful facial expressions but occur following emotional auditory stimuli (Zeelenberg \& Bocanegra, 2010), fear-conditioned stimuli (Lee, Baek, Lu, \& Mather, 2014a; Lojowska, Gladwin, Hermans, \& Roelofs, 2015) and emotional images (Song \& Keil, 2013).

\section{Affecting Speed and Accuracy in Perception}

Why does emotion both enhance and impair vision? According to the Affecting Speed and Accuracy in Perception account (ASAP; Bocanegra, 2014) emotion induces an inhibitory interaction between the magnocellular-type (M-type) and parvocellular-type (P-type) channels in the visual system. M-type channels respond quickly to coarse-grained spatial (LSF) information whereas P-type channels exhibit a slower, more sustained response to fine-grained spatial (HSF) information (Breitmeyer \& Williams, 1990; Callaway, 1998). Emotion is thought to potentiate the fast-acting M-type channels leading to increased sensitivity to LSF information while concurrently inhibiting the slower-acting parvocellular-type (P-type) channels leading to poorer sensitivity to HSF information.

A challenge for the ASAP account and other accounts of the effects of emotion cues on attention and perception is accounting for a diverse effects of emotion cues on both RTs and accuracy rates. For example, emotion cues slow RTs in some experiments (e.g., Fox, Russo, \& Dutton, 2002; Hodsoll, Viding, \& Lavie, 2011) and speed RTs in others (e.g., Becker, 2009; Quinlan \& Johnson, 2011), and also, improve and reduce accuracy in separate experiments (Sutherland \& Mather, 2012). A notable feature of the ASAP account is that it takes a broader perspective by attempting to provide a framework for interpreting the effects of emotion stimuli on both RT and accuracy rates. The ASAP account specifies when emotion stimuli will affect RTs or accuracy depending on whether the task relies more on processing via either the P-type channel (for fine grained analyses) or the M-type channel (for coarse-grained analyses). For example, if a task requires identification and fast responses (typically required for RT paradigms involving a single stimulus) then the prediction is that emotion will slow-down RTs because identification relies relatively more on the slower and more sustained activation of the P-channel (Bocanegra, 2014). However, for tasks that require identification and emphasize slower, accurate responses (typically required for multi-stimulus tasks identification) then the claim is that emotion will increase the response duration of the P-type channel leading to greater accuracy. This basic model was able to explain the results of multiple experiments in which participants judged faces with different spatial frequency information removed. For example, in support of the idea that the emotion modulates the P-channel (required for identification) one experiment (Experiment 1c; Bocanegra, 2014) showed that RTs were reliably slower for fearful (vs neutral) expressions when people were asked to judge the sex of a single fearful face with low-spatial frequency information removed. Overall, the ASAP account; 1) offers a compelling account of the dissociable effects of emotion on high and low spatial frequency information and 2) argues for a direct effect of emotion on vision - emotion modulates visual input prior to the attentional system (Bocanegra, 2014). 
Evaluation. Beyond the results of experiments that have manipulated spatial frequency information it is not clear how the ASAP account might explain the effects of emotional stimuli on RT and accuracy in experiments that have neither controlled for nor manipulated the presence of spatial frequency information. For facial expressions specifically, experiments have reported a diverse set of findings including: 1) reductions in target detection accuracy following expressions presented in rapid sequence of images (de Jong, Koster, van Wees, \& Martens, 2010; Maratos, 2011; Peers \& Lawrence, 2009; Sigurjónsdóttir, Sigurðardóttir, Björnsson, \& Kristjánsson, 2015; Stebbins \& Vanous, 2015; Vermeulen, Godefroid, \& Mermillod, 2009) 2) increased target detection accuracy following fearful compared to angry expressions (Taylor \& Whalen, 2014) 3) faster visual search for complex images following the presentation of a fearful face (Becker, 2009; Quinlan \& Johnson, 2011) 4) slower RTs when fearful and other expressions are presented as task-irrelevant distractors (Berggren, Richards, Taylor, \& Derakshan, 2013; Fox et al., 2002; Georgiou et al., 2005; Gupta, Hur, \& Lavie, 2016; Hodsoll et al., 2011; Van Dillen \& Koole, 2009) and 5) faster RTs for neutral probes following in the same location as a threat-related expression (e.g., Bradley, Mogg, Falla, \& Hamilton, 1998). Although it is difficult to summarize the interpretation for each result (for reviews see; Mogg \& Bradley, 2016; Yiend, 2010) a common interpretation of both RT and accuracy effects in these studies is that emotion biases the competition for limited resources a competition that emotion stimuli are, as the result of evolutionary pressures, predisposed to win or influence.

\section{The speed-accuracy trade-off}

The many studies of emotion on attention and perception have tended to overlook a fundamental aspect of decision making under time pressure namely, the speed-accuracy trade-off; the ubiquitous finding that accuracy of a decision varies with the time taken to make a decision speed correlates with accuracy (Schouten \& Bekker, 1967; Wickelgren, 1977). The majority of previous studies into the effects of emotion on attention have not directly tested for an effect of emotion on the relationship between accuracy and RTs and therefore, we do not know to what extent the results might reflect a speed-accuracy trade-off. Intuitively though they might do. For example, fearful expressions are thought to warn of potential threat - a warning signal (Davis et al., 2011; Taylor \& Whalen, 2014; Whalen, 1998) - therefore, responding cautiously by slowing down to avoid a mistake (trading speed for accuracy) is an intuitively plausible response to fearful expressions. For other emotional stimuli a speed-accuracy trade-off might lead to a different pattern. For example, people might dwell on emotionally arousing images - spend longer looking at them - and consequently they might rush a response when the target image appears - they trade accuracy for speed. In sum, the speed-accuracy trade-off is fundamental aspect of choice behaviour that has received little attention in research studies testing for the effects of emotion on perception and attention.

\section{Diffusion Model}

Distinguishing the effects of emotion on caution on the one hand and perception on the other is challenging using a single measure such as reaction time because it is difficult to determine whether a pattern of RTs or accuracy rates reflects either caution or an effect of emotion on either perception or attention. The Diffusion Model (Ratcliff, 1978; Ratcliff \& McKoon, 2008) includes model parameters that index both caution and evidence accumulation and therefore, is a suitable way of determine if emotion affects caution, perception or both. It does this by modelling the distribution of RTs and both correct and incorrect responses in terms of separate, latent psychological processes. 
- - - - - Insert Figure 1 here - - - - -

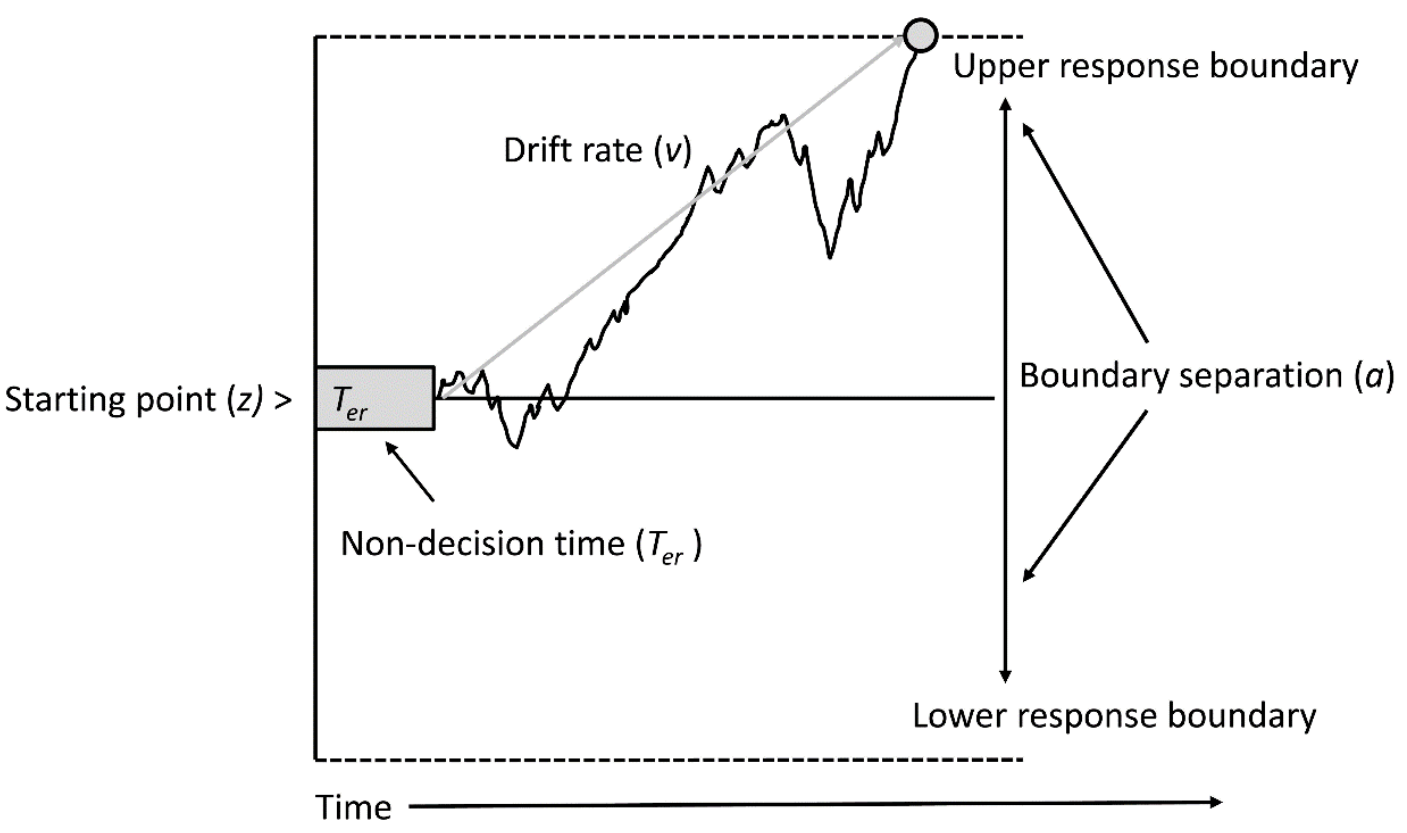

Figure 1. The Diffusion Model (Ratcliff, 1978; Ratcliff \& McKoon, 2008). Evidence accumulation begins at a starting point $\mathrm{z}$, and proceeds toward absorbing boundaries that determine when the decision criterion is met and the accumulation process ends. The drift rate indicates how fast information accumulates toward a boundary. Non-decision times are modelled as the additional time needed for processing before and after the accumulation process. Non-decision time includes the time to encode the stimulus and prepare a response. Further parameters can also be included in the model (e.g., trial-by-trial variability in the starting point parameter $\mathrm{z}$ ).

One way of understanding the Diffusion Model is to compare it to Signal Detection Theory (Macmillan \& Creelman, 2005). The Diffusion Model extends signal detection theory by modelling signal detection as a dynamic process of evidence accumulation - the repeated sampling of information until a criterion has been reached. A graphic representation of the accumulation process and the 4 main Diffusion Model parameters is shown in Figure 1. To illustrate the Diffusion Model, consider the orientation detection task used previously to test for the effects of emotion on perception (Nicol et al., 2013) in which a person must detect whether a tilted Gabor patch is present or absent. On each trial evidence is repeatedly sampled until the accumulated evidence favours either the present or absent criteria. If the observer has particularly good vision or the stimulus is high contrast - then sensory information will accumulate rapidly toward the criteria for "tilt absent" or "tilt present". The decision criteria for the "tilt present" and "tilt absent" responses are modelled as absorbing boundaries that determine when the criterion is reached and the accumulation process ends. Larger separation between the boundaries indicates greater caution because the person requires more evidence before reaching a decision. A narrow boundary separation indicates more liberal decision making. Finally, the Diffusion Model includes 2 further model parameters: 1) a starting point parameter (z), and 2) a parameter that indexes non-decision times ( $\mathrm{t}$ or Ter). The starting point parameter $(\mathrm{z})$ is an index of the starting point of the drift diffusion process. If the starting point parameter is higher in one condition, then this may represent a response bias toward one of the response options. Non-decision times (Ter) models all processes occurring before or after the decision for example, encoding processes (before) and response execution processes (after). 


\section{Current research}

Here, I used the Diffusion Model to test the idea that seeing facial expressions influences visual perception and also, decision making caution. To test these hypotheses, I used a modified version of a task used in previous research (Bocanegra \& Zeelenberg, 2009b; Borst \& Kosslyn, 2010b; Nicol, Perrotta, Caliciuri, \& Wachowiak, 2013b). Specifically, participants detected a tilted HSF Gabor patch that appeared after the brief presentation of a facial expression. I wished to minimize the influence of both shifts in visual attention and decision making noise (Dosher $\& \mathrm{Lu}$, 2000) and therefore, following other researchers (Lee, Baek, Lu, \& Mather, 2014b) all stimuli were presented in single, central location.

Predictions. According to ASAP account, emotion will slow-down RTs when participants are required to judge a single HSF stimulus. For the Diffusion Model, the prediction is that impaired perception will be reflected in a reduction in the drift rate. Also, if people respond to fearful facial expressions as warning signals (Whalen, 1998) by becoming more cautious then the second prediction is that Diffusion Model parameter that measures caution - boundary separation - will be higher following fearful compared to neutral facial expressions.

\section{Hierarchical Drift Diffusion Modelling and data analyses}

I used Hierarchical Drift Diffusion Modelling (Vandekerckhove, Tuerlinckx, \& Lee, 2008, 2011b; Wiecki et al., 2013) to isolate the psychological processes responsible for the effects of facial expressions on visual perception. Hierarchical Bayesian estimation is particularly suited to studies with relatively small number of observations $(<48)$ per cell of the design because subject and group-level posterior estimates can reciprocally influence each other leading to greater statistical precision. A further more general advantage of Bayesian estimation is that it is possible to directly quantify uncertainty for a given experimental hypothesis by comparing the overlap between the (posterior) distributions of specific parameter estimates - a true confidence or credibility interval for an effect.

Priors. In the Bayesian statistical approach the calculation of plausible values for specific model parameters requires the specification of priors. The HDDM toolbox includes informative priors to constrain estimates of the model parameters. The priors are based on analyses of 23 published studies (Matzke \& Wagenmakers, 2009) and I have used these in the current study.

Convergence. HDDM is a Bayesian modelling approach that uses Markov Chain Monte Carlo (MCMC) simulations to estimate a range of plausible values for specific model parameters. MCMC chains were composed of 200 samples as a burn-in (to increase chain stability) and 4 chains of 5000 iterations to estimate the posterior distribution of each parameter. Checks for chain convergence included both visual inspection of MCMC chains, histograms and autocorrelation plots and calculation of the R-hat (Gelman-Rubin) statistic. Convergence check plots for each experiment and also, the Gelman-Rubin statistic for each effect can be found in the online supplement. Also, in the online supplement I have included example code and the raw data for Experiment 4.

Model Selection. I used the Deviance Information Criterion (DIC; Spiegelhalter, Best, Carlin, \& Van Der Linde, 2002) as a criterion for assessing model fit. Although smaller DIC values indicate a better fit the reduction needs to be sufficiently large to justify an increased complexity. Reductions in the DIC greater than 5 are considered substantial ('DIC', n.d.) and therefore, I used a reduction in the DIC of 5 or more as the criteria for judging improved model fit and selecting a more complex model. Also in the online supplement (Sections 5 and 6) I have carried out an assessment of the quality of the model fit by simulating data from the posterior distribution of each fitted model and subsequently plotting the simulated (estimated) data against the observed data. This is a standard approach to quality assessment in Bayesian parameter estimation and is referred to as a posterior predictive check (Gelman et al., 2014).

All models included person-specific estimates for the Ter (non-decision times), $a$ (boundary separation), $v$ (drift rate) and $z$ (starting point) parameters. These parameters were estimated as draws from a group-level distribution centred on a mean $\mu$ and variance $\sigma^{2}$. For example, a specific person's boundary separation parameter value $(\alpha)$ was estimated from a group-level normal distribution of boundary separation values with $\mu_{\alpha}$ and variance $\sigma_{\alpha}^{2}$. This means that the grouplevel parameters (e.g., the overall effect of fearful expressions across individuals) are adjusted for 
individual differences. The group-level varying parameters are the parameters of interest and therefore, model selection was carried out comparing DIC values for model variants in which group-level parameters were either fixed or allowed to vary by expression.

Initially, for each experiment, I tested eight different models in which the three parameters of interest $(v, a, \mathrm{Ter})$ were either fixed or allowed to vary by expression across the eight model variants. In the empty model, all three parameters $(v, a$, Ter $)$ were fixed across levels of expression. For each experiment, allowing the drift rate to vary by expression failed to improve model fit (as assessed by the lower DIC value relative to other models). Further testing was conducted to establish the validity of the initial model selection. Specifically, 2 further models were estimated (for each experiment) in which drift rates were allowed to vary as a function of degree of tilt of the Gabor patch and both degree of tilt and expression. This additional modelling was conducted to establish whether the modelling was sensitive to the effect of degree of tilt on drift rates and also, to establish whether adding degree of tilt might improve the ability to detect and effect of expression on drift rates. For all experiments, allowing the drift rates to vary as a function of degree of tilt improved model fit -DIC value was reduced. However, allowing drift rates to vary as a function of expression in addition to degree of tilt failed to lower the DIC value and therefore, for all final models degree of tilt for drift rates. A table of the DIC values for each experiment can be found in the online supplement (Section 3).

Sample Size and Treatment of RT data. The design is based on a similar study (Nicol et al., 2013) with a similar number of observations per cell of the design. Nicol et al tested 20 participants and recorded a mean reduction in orientation sensitivity due to fearful vs neutral expressions of .3. Although I used Bayesian analyses in this research, a frequentist power analyses (with correlation between measures set to .6) indicates that 28 participants is needed to replicate this effect.

For treatment of RT data, responses that were either faster than $100 \mathrm{~ms}$ or slower than $3000 \mathrm{~ms}$ were removed prior to modelling. This was carried out because Diffusion Modelling estimates can be biased by fast responses (Voss, Voss, \& Lerche, 2015) and also, because the Hierarchical Drift Diffusion Modelling software (Wiecki et al., 2013) requires RTs greater $100 \mathrm{~ms}$ in duration to find initial sampling values.

Mean RT and Signal Detection Analyses. To enable a comparison with previous research, both mean RTs and the signal sensitivity parameter $d^{\prime}$ were calculated for each participant for each expression type (fearful, neutral). The signal sensitivity parameter $d^{\prime}$ was calculated using the standard formula: $\mathrm{z}$ (hits) $-\mathrm{z}$ (false alarms) (Stanislaw \& Todorov, 1999). The resulting mean RTs and mean d' indices for each subject and each expression type were analysed in 2 separate Bayesian Linear Mixed Models. Modelling was carried out in the Stan modelling language (Carpenter et al., in press) using the rstanarm package (Gabry et al., 2016) as an interface between Stan and R (R Core Development Team, 2013). Weakly informative priors (the default for rstanarm) were used for all tests. All models included random intercepts for participants. MCMC chains were composed of 200 samples as a burn-in (to increase chain stability) and 2 chains of 5000 iterations each. All models converged.

\section{Experiment 1}

The aim of Experiment 1 is to establish whether people will also react cautiously to fearful expressions when they are asked to judge HSF target stimuli.

\section{Participants}

\section{Method}

Twenty-eight psychology students from the Leeds Beckett University took part in the study in return for a course credit. There were 4 males (mean age $=20$ ) and 24 females (mean age $=23$ ). All participants provided written informed consent and received course credit for their participation. The experimental protocol was approved by the Department of Psychology Ethics Committee of the Leeds Beckett University.

\section{Stimuli and Apparatus}

The face stimuli were 6 digitized photographs were selected from the Ekman and Friesen pictures of facial affect (Ekman \& Friesen, 1976). The photographs were of 1 male and 2 females each displaying a fearful and neutral facial expression. The target stimulus was a high spatial 
frequency Gabor patch (sinusoidal grating enveloped by a Gaussian mask; 6 cycles per degree) created using the python libraries packaged with PsychoPy (Peirce, 2007). The Gabor patch was either upright or angled equally often either 1, 2 or 3 degrees to the left or right. At a viewing distance of $60 \mathrm{cms}$ the Gabor patch subtended $5.5^{\circ} \times 5.5^{\circ}$ of visual angle. Stimulus presentation and data collection were controlled by PsyToolkit (Stoet, 2010).

\section{Design and Procedure}

Participants completed 16 practice trials followed by 384 trials in a single block. On half the trials the Gabor patch was upright and on the remaining (192) trials the patch was angled (equally often) either 1, 2 or 3 degrees to the left or right. Trials were comprised of balanced factorial combinations of face identity and expression. In other words, every possible target type (e.g., a Gabor patch titled 1 degree to the left) was equally likely to appear after every possible face type (e.g., a male face displaying fear).

All stimuli were presented in the center of gray background (RGB, 128,128,128). Every trial contained the following sequence of events with each event displayed for a specific duration (given in parentheses): 1) fixation cross (500 $\mathrm{ms}), 2)$ face $(500 \mathrm{~ms}), 3)$ upright or tilted Gabor patch (75 $\mathrm{ms}$ ) 4) response interval (until response or $3000 \mathrm{~ms}$ ) and 5) feedback (500 ms). Participants were told to: 1) start by looking at the fixation cross 2) look at the face and 3) press either the " $\mathrm{m}$ " key if the set of lines were tilted (either left or right) or "z" key if the lines were upright (not tilted). Visual feedback was given if a response was not given within 3 seconds ("too slow") and also, for both correct ("correct"), incorrect ("error") responses.

Signal Detection and RT analyses. Mean RTs were slower following fearful $(M=1095$, $S D=409)$ compared to neutral $(M=1062, S D=431)$ expressions, $\beta=32.7,95 \%$ CrI [14, 52]. For mean d-prime scores the effect of expression was not significant - the $95 \%$ Credibility Interval for difference between neutral $(M=.92, S D=0.70)$ and fearful $(M=.87, S D=0.72)$ expressions included zero, $\beta=-0.1,95 \% \mathrm{CrI}[-0.2,0.1]$.

\section{HDDM results}

Initial model comparison of the DIC values across the 8 model variants showed that in the best-fitting model both boundary separation values and non-decision times were allowed to vary as a function of expression type. Allowing drift rates to vary as a function of degree of tilt improved model fit. Allowing drift rates to additionally vary as a function of expression did not lead to further improvements in model fit.

\section{- - - - - -Insert Figure 2 here - - - . - -}

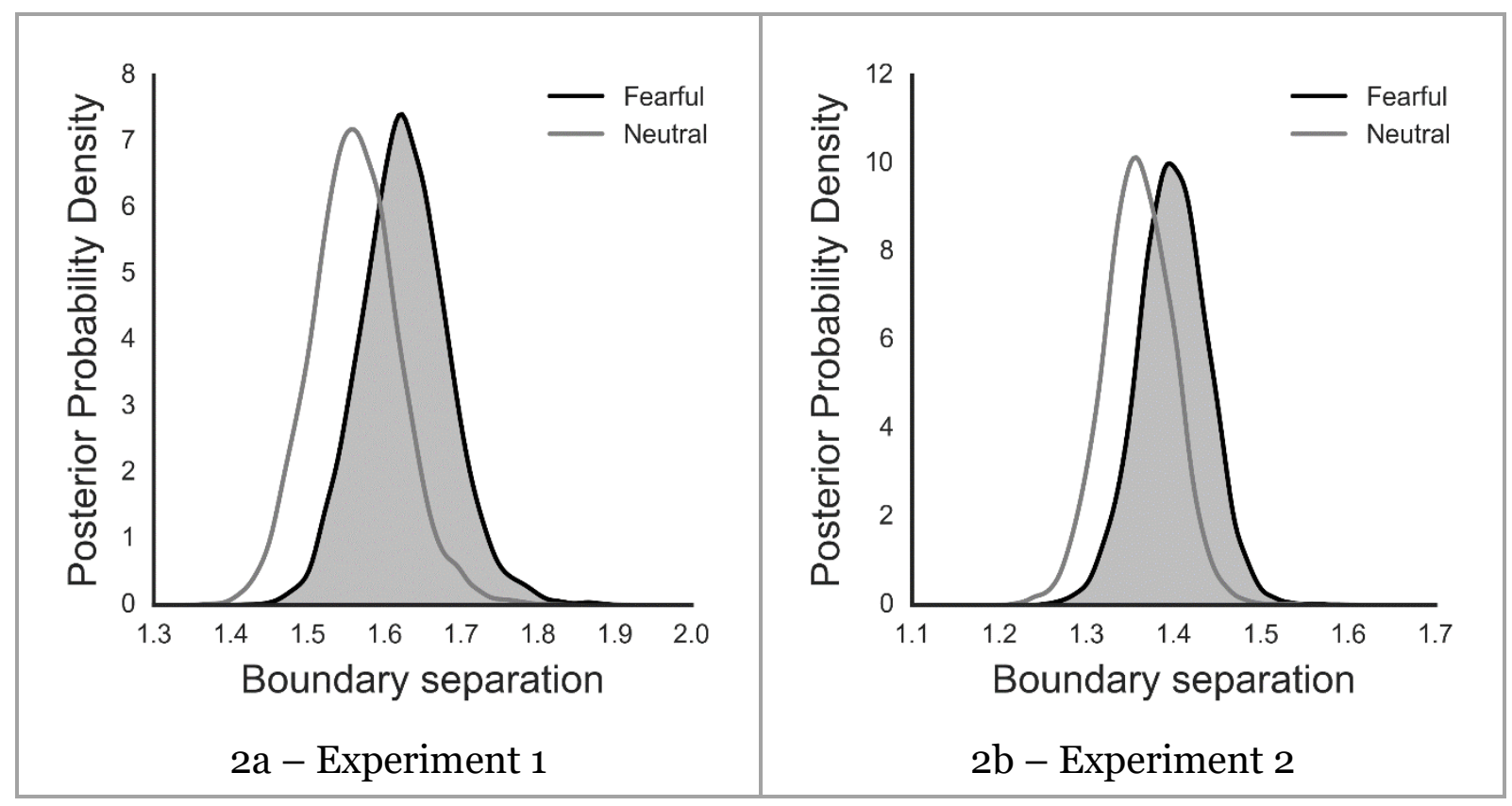

Figure 2-Posterior distributions for the boundary separation parameter for the fearful and neutral face conditions for Experiment 1 (2a) and Experiment 2 (2b), separately. 


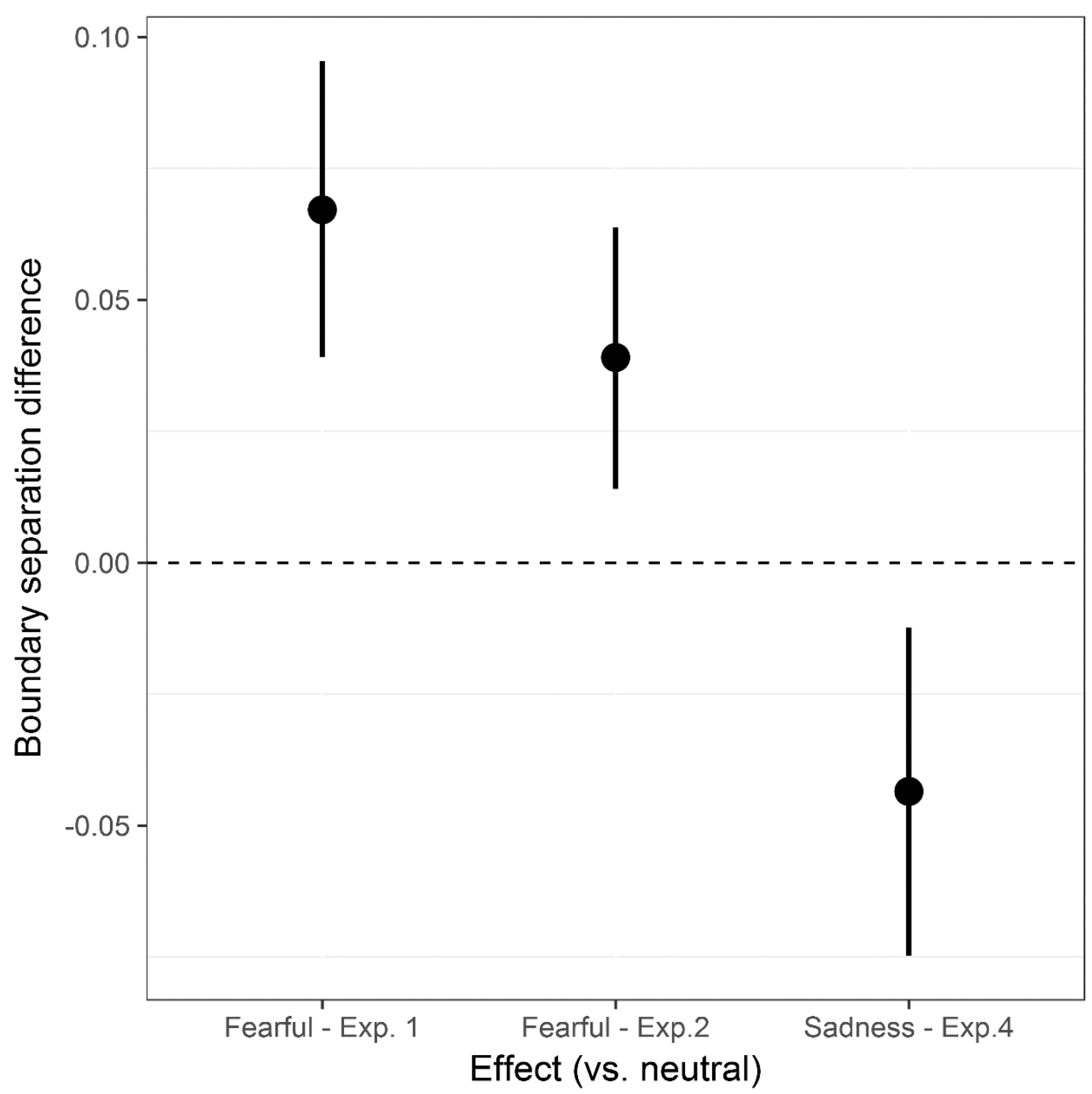

Insert Figure 3 here - - - - - -

Figure 3 - Mean estimated effect of expression on boundary separation values for Experiments 1 (fearful vs neutral), 2 (fearful vs neutral) and 4 (sadness vs neutral). Positive values indicate increased caution. Error bars are 95\% Credibility intervals for the estimated effect. A difference of zero is represented as a dashed horizontal line at zero on the y axis.

Boundary separation. Regression analyses of boundary separation supports the hypothesis that people increase decision making caution following fearful expressions. As shown in Figure 2a mean posterior estimates of boundary separation values were higher following fearful compared $(M=1.62 ; S D=0.07)$ compared to neutral expressions $(M=1.56 ; S D=0.05)$. The boundary separation effect for fearful expressions is shown in Figure 3 where it can be see that the posterior distribution for the increase in boundary separation for fearful (vs neutral) expressions did not contain zero, $\beta=0.06,95 \% \mathrm{CrI}=0.03$ to 0.09 .

Non-decision times. Regression analyses of non-decision times showed that nondecision times decreased following fearful expressions. Specifically, mean posterior estimates of non-decision times were slightly lower following fearful compared $(M=.50)$ compared to neutral expressions $(M=.51)$. The posterior distribution for the decrease in non-decision time for fearful (vs neutral) expressions did not contain zero, $\beta=-0.006,95 \% \mathrm{CrI}=-0.01$ to -0.001 .

Drift rates. Regression analyses showed that drift rates were lowest for Gabor patches titled 1 degree $(M=-0.01, S D=0.11)$ compared to all other conditions: 2 degrees $(M=0.63, S D=$ 
0.11), $\beta=0.64,95 \%$ CrI $[0.55,0.73], 3$ degrees $(M=0.95, S D=0.11), \beta=0.96,95 \%$ CrI $[0.87$, 1.05] and o degrees $(M=0.77, S D=0.10), \beta=0.78,95 \% \mathrm{CrI}[0.70,0.85]$.

\section{Discussion}

Results support the hypothesis that people react cautiously following exposure to fearful expressions. Mean RTs were slower and values of the Diffusion Model parameter that indexes caution (boundary separation) were higher for fearful compare to neutral expressions. The modelling results matter because a different conclusion might have been reached based solely on standard statistical analyses of either mean RTs. According to ASAP account (Bocanegra, 2014) emotional expressions slow down RTs in single-stimulus tasks that require analyses of HSF information. RT slowing was recorded but the modelling results suggest increased caution and not impaired perception underpins this behavioural effect. This is because Diffusion Modelling indicated that the effects of fearful expressions are best explained as reflecting differences in the boundary separation model parameter (representing increased caution) rather than differences in the drift rate (representing evidence accumulation). There was also a reduction in non-decision times following fearful compared to neutral expressions. This effect was not predicted and the effect was small (-0.006) and was not replicated in Experiment 2, and therefore, this effect is not discussed further.

\section{Experiment 2}

In Experiment 1, fearful expressions led to increased caution and reduced non-decision times. A key difference between the results of Experiment 1 and those reported in previous research is that mean signal sensitivity $d^{\prime}$ was lower $(M=.89)$ than that used in previous studies (e.g., Bocanegra \& Zeelenberg, 2009). Experiment 2 was an attempt to replicate the results of Experiment 1 using an easier task. Specifically, in Experiment 2 the angle of Gabor patch varied (on target present trials) from 2 to 4 degrees rather than the 1 to 3 degrees used in Experiment 1. Also, the number of face stimuli was increased to establish whether the effect generalises across different stimuli.

\section{Participants}

\section{Method}

Twenty-eight psychology students from Leeds Beckett University took part in the study in return for a course credit. There were 7 males (mean age $=21$ ) and 21 females (mean age $=20$ ). All participants provided written informed consent and received course credit for their participation. The experimental protocol was approved by the Department of Psychology Ethics Committee of the Leeds Beckett University.

\section{Stimuli and Apparatus}

The face stimuli were 12 digitized photographs also selected from the Ekman and Friesen pictures of facial affect. The photographs were of three males and three females each displaying a fearful and neutral facial expression. The target stimuli were the same high spatial frequency Gabor patches (6 cycles per degree) used in Experiment 1, that were either upright or angled either 2, 3 or 4 degrees to the left or right.

\section{Design and Procedure}

Participants completed 16 practice trials followed by 288 trials in a single block. Of the 288 trials in the main block half contained a tilted target and half contained an upright target (absent trials). On the 144 target present trials the lines were angled (equally often) either 2, 3 or 4 degrees to the left or right. Fearful and neutral expressions were equally likely to precede either an upright Gabor patch or a tilted Gabor patch. All other details of the experiment were identical to that used in Experiment 1.

\section{Results}

Mean RTs were again slower following fearful $(M=554, S D=203)$ compared to neutral $(M$ $=540, S D=199)$ expressions, $\beta=32$, 95\% CrI $[14,52]$. For mean d-prime scores the effect of expression was not significant - the 95\% Credibility Interval for difference between neutral $(M=$ $1.81, S D=0.85)$ and fearful $(M=1.79, S D=0.89)$ expressions included zero, $\beta=-0.1,95 \%$ CrI [$0.02,0.12]$. 


\section{HDDM results}

Model selection. The model in which the boundary separation value was free to vary as a function of the fixed effect of expression had the lowest DIC value (666) compared to either the null model (DIC $=669$ ) or models in which either drift rates (DIC $=668$ ) or non-decision times $(\mathrm{DIC}=669)$ were free to vary as a function of expression. Although the model in which both drift rates and boundary separation values were free to vary had the lowest DIC value (DIC = 665) the decrease did not reach the criteria of a DIC of 5 or more. Allowing drift rates to vary as a function of degree of tilt improved model fit (DIC $=167$ ). Allowing drift rates to additionally vary as a function of expression did not lead to further improvements in model fit (DIC = 171).

Boundary separation. Regression analyses of boundary separation once again supports the hypothesis that people increase decision making caution following fearful expressions. As shown in Figure $2 b$ mean posterior estimates of boundary separation values were higher following fearful compared $(M=1.4, S D=0.04)$ compared to neutral expressions $(M=1.36, S D=0.04)$. The boundary separation effect for fearful expressions is shown in Figure 3 where it can be see that the posterior distribution for the increase in boundary separation for fearful (vs neutral) expressions did not contain zero, $\beta=0.04,95 \% \operatorname{CrI}[0.01,0.06]$.

Drift rates. Regression analyses showed that drift rates were lowest for Gabor patches titled 2 degrees $(M=1.06, S D=0.18)$ compared to all other conditions: 3 degrees $(M=1.94, S D=$ 0.18) , $\beta=0.88,95 \% \operatorname{CrI}[0.75,1.01], 4$ degrees $(M=2.35, S D=0.18), \beta=1.29,95 \% \operatorname{CrI}[1.16$, 1.42] and o degrees $(M=2.14, S D=0.17), \beta=1.08,95 \%$ CrI $[0.98,1.18]$.

\section{Discussion}

Results once again support the idea that people react cautiously to seeing fearful expressions because the Diffusion Model parameter representing caution was higher for fearful compared to neutral expressions. In contrast to Experiment 1, non-decision times did not differ between expression types.

\section{Experiment 3}

So far, results support the idea that people react cautiously to fearful expressions - they interpret fearful expressions as warning signals. However, this conclusion is based on a comparison between fearful and neutral facial expressions and consequently, it remains possible that the effect could be due to perceiving anything expressive, negative or emotional. Experiment 3 addresses this issue by replacing fear with happiness (Experiment 3) and in a separate experiment, with sad facial expressions (Experiment 4).

\section{Participants}

\section{Method}

Twenty-eight psychology students from Leeds Beckett University took part in the study in return for a course credit. There were 3 males (mean age $=19$ ) and 25 females (mean age $=20$ ).

\section{Stimuli, design and procedure}

The method was identical to Experiment 2 except that fearful expressions were replaced by happy expressions of the same individuals from the same stimulus set. All participants provided written informed consent and received course credit for their participation. The experimental protocol was approved by the Department of Psychology Ethics Committee of the Leeds Beckett University.

\section{Results and Discussion}

The effects of expression was not significant for analyses of either RTs or $d^{\prime}$. Specifically, the 95\% Credibility Interval for differences in mean RTs following happy $(M=570, S D=225)$ compared to neutral $(M=568, S D=210)$ included zero, $\beta=2.3,95 \%$ CrI $[-5,5]$. Similarly, the 95\% Credibility Interval for differences in the $d^{\prime}$ scores following happy $(M=1.98, S D=0.70)$ compared to neutral $(M=1.96, S D=0.77)$ expressions also included zero, $\beta=0.01,95 \%$ CrI [$0.15,0.07]$.

\section{HDDM results}

None of the 8 HDDM models that allowed specific model parameters to vary as a function of facial expression improved model fit compared to the empty model. Further testing showed that modelling drift rates to vary as a function of degree improved model fit compared to the empty to model. Adding expression to the latter model did not improve model fit. 
Drift rates. Regression analyses showed that drift rates were lowest for Gabor patches titled 2 degrees $(M=1.25, S D=0.16)$ compared to all other conditions: 3 degrees $(M=1.95, S D=$ 0.16) , $\beta=0.69$, 95\% CrI [0.57, 0.81] , 4 degrees $(M=2.32, S D=0.16), \beta=1.06,95 \%$ CrI [0.93, 1.19] and o degrees $(M=2.01, S D=0.15), \beta=0.75,95 \% \operatorname{CrI}[0.65,0.85]$.

\section{Experiment 4}

In Experiment 3 both standard traditional statistical analyses and the Diffusion Modelling results failed to support of the claim that increased caution is simply due to presenting a face expressing emotion in general. Nonetheless, it remains possible that seeing a negative expression rather than an expression associated with threat might also elicit a cautionary response. To test this idea, the happy expressions used in Experiment 4 were replaced with sad facial expressions. Sad expressions are sometimes perceived a request for help or comfort (Horstmann \& Gernot, 2003) and therefore, people are not expected to respond to sad expression with increased caution.

\section{Method}

\section{Participants}

Twenty-eight psychology students from Leeds Beckett University took part in the study in return for a course credit. There were 5 males (mean age $=20$ ) and 23 females (mean age $=19$ ). All participants provided written informed consent and received course credit for their participation. The experimental protocol was approved by the Department of Psychology Ethics Committee of the Leeds Beckett University.

\section{Stimuli, design and procedure}

The method was identical to Experiment 3 except that happy expressions were replaced with photos of sad expressions of the same individuals from the same stimulus set.

\section{Results}

The effects of expression was not significant for analyses of either RTs or $d^{\prime}$. Specifically, the 95\% Credibility Interval for differences in mean RTs following sad $(M=560, S D=120)$ compared to neutral $(M=560, S D=121)$ included zero, $\beta=-0.02$, 95\% CrI $[-7,7]$. Similarly, the $95 \%$ Credibility Interval for differences in the $d^{\prime}$ 'scores following $\operatorname{sad}(M=1.76, S D=0.99)$ compared to neutral $(M=1.91, S D=1.05)$ expressions also included zero, $\beta=-0.15,95 \% \mathrm{CrI}[-0.37,0.07]$.

\section{HDDM results}

Initial model comparison of the DIC values across the 8 model variants showed that in the best-fitting model both boundary separation values and non-decision times were allowed to vary as a function of expression type. Allowing drift rates to vary as a function of degree of tilt improved model fit. Allowing drift rates to additionally vary as a function of expression did not lead to further improvements in model fit.

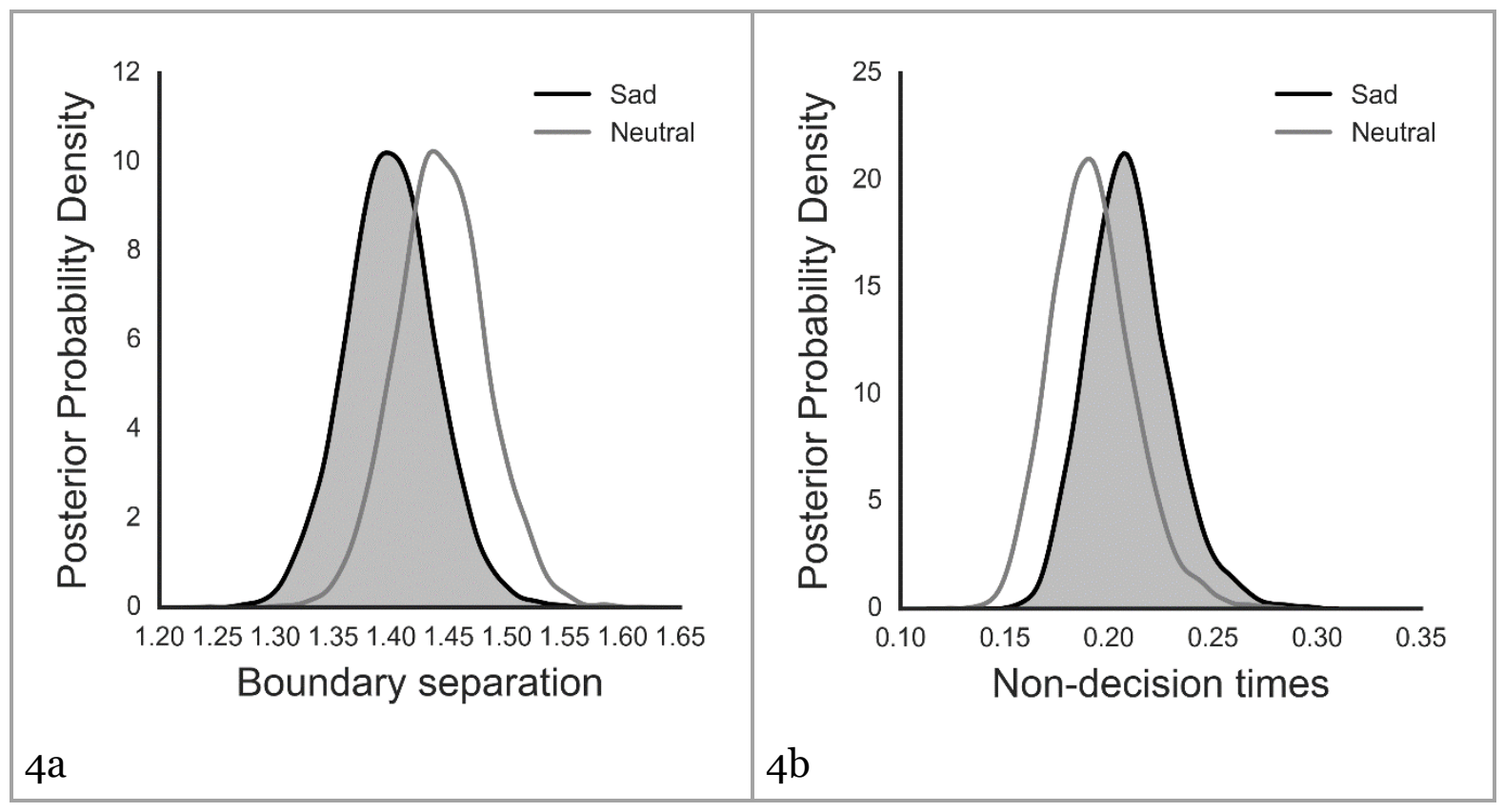


- - - - - -Insert Figure 4 here - - - - - -

Figure 4-Posterior distributions for the boundary separation parameter (4a) and non-decision times (4b) for the sad and neutral face conditions for Experiment 4.

Boundary separation. Regression analyses of boundary separation supports the hypothesis that decision making caution decreases following the presentation of sad expressions. As shown in Figure 4a mean posterior estimates of boundary separation values were lower following sad compared $(M=1.40, S D=0.04)$ compared to neutral expressions $(M=1.44, S D=$ 0.02). The boundary separation effect for sad expressions is shown in Figure 3 where it can be see that the posterior distribution for the increase in boundary separation for sad (vs neutral) expressions did not contain zero, $\beta=-0.04,95 \% \mathrm{CrI}[-0.07,-0.01]$

Non-decision times. Regression analyses of non-decision times showed that nondecision times increased following sad expressions. As shown in Figure 4b, mean posterior estimates of non-decision times were higher following sad expressions compared $(M=.21, S D=$ 0.02) compared to neutral expressions $(M=.19, S D=0.02)$. The posterior distribution for the increase in non-decision time for sad (vs neutral) expressions did not contain zero, $\beta=0.017,95 \%$ CrI [0.01, 0.02]

Drift rates. Regression analyses showed that drift rates were lowest for Gabor patches titled 2 degrees $(M=0.94, S D=0.16)$ compared to all other conditions: 3 degrees $(M=1.75, S D=$ 0.16) , $\beta=0.81,95 \% \operatorname{CrI}[0.69,0.93], 4$ degrees $(M=1.96, S D=0.16), \beta=1.02,95 \% \operatorname{CrI}[0.89$, 1.14 ] and o degrees $(M=1.84, S D=0.16), \beta=0.90,95 \% \operatorname{CrI}[0.80,0.99]$.

\section{Discussion}

Decision making caution decreased following sad expressions - participants were more liberal - the opposite pattern to that recorded for fearful expressions. Moreover, non-decision times were slower for sad compared to neutral expression - again, the opposite pattern to that recorded for fearful expressions (in Experiment 1).

These effects were not expected and therefore, given the possibility of the effect being due a noisy sample (Nosek, Spies, \& Motyl, 2012) I increased the sample size by adding a further 18 participants $(\mathrm{N}=46)$. I re-estimated the HDDM model in which boundary separation values and non-decision times were allowed to vary as a function of expression type and also, the model in which all 3 Diffusion Model parameters were allowed to vary as a function of expression. The model with 2 parameters remained the best fit ( $\mathrm{DIC}=3656$ ) compared to the 3 parameter model (DIC $=3660)$ and again, for the larger sample, non-decision times were slower following sad $(M=$ $.20, S D=0.01)$ compared to neutral expressions $(M=.19, S D=0.01), \beta=0.01,95 \% \mathrm{CrI}=0.07$ to 0.01 and also, the boundary separation values were lower for sad $(M=1.36, S D=0.03)$ compared to neutral expressions $(M=1.38, S D=0.03), \beta=-0.02,95 \% \mathrm{CrI}=-0.05$ to -0.005$)$. Also, the effects expression on boundary separation $(\beta=-0.02,95 \% \mathrm{CrI}=-0.05$ to -0.002$)$ and nondecision times ( $\beta=0.01,95 \% \mathrm{CrI}=0.007$ to 0.015$)$ remained after including the degree of tilt of the Gabor patch as a predictor in the regression model. Finally, analyses of $d^{\prime}$ scores for the larger sample indicated a small decrease in $d$ ' following sad compared to neutral expressions, $\beta=-0.14$, 95\% CrI [-0.29, -0.008].

\section{General Discussion}

Hierarchical Drift Diffusion Modelling provided several novel insights into perceptual decision making following the presentation of facial expressions. In support of the idea that fearful expressions warn of potential threat (Davis et al., 2011; Whalen, 1998), Diffusion Modelling results indicate that people respond cautiously following fearful expressions. Specifically, Hierarchical Drift Diffusion Modelling showed that the model parameter that describes response caution boundary separation - was higher after exposure to fearful compared to neutral facial expressions. This effect was not recorded for either happy sad expressions. For sad expressions boundary separation values were lower and non-decision times were longer.

A key implication of the modelling results is that slower RTs to targets following fearful facial expressions need not necessarily reflect an impairment or processing cost. Instead, the results show that such a pattern might reflect increased decision making caution. Studies have 
typically interpreted RT slowing due to facial expressions as reflecting a processing cost and subsequently concluded that effects reflect an effect of emotion on either attention or perception. For example, a recent study found that RTs were reliably slower for fearful (vs neutral) expressions in one specific experiment in which people were asked to judge the sex of a single fearful with lowspatial frequency information removed (Experiment 1c; Bocanegra, 2014). Based on this result it was concluded that emotion affects early visual processes by decelerating the onset latency of the P-type channel. I also recorded RT slowing for high spatial information following fearful expressions. However, Diffusion Modelling using HDDM supports the conclusion that RT slowing was due to increased decision making caution rather than impaired perception. Specifically, in Experiment 1 and Experiment 2 boundary separation values were higher following fearful compared to neutral facial expressions but there was no evidence that fearful expressions impaired perception by reducing information accumulation (lowering drift rates).

A further implication is that models of how emotion affects visual perception may now need to consider a greater role for high-level decision making. In current models of emotion and attention high level influences are typically considered in terms of the task set or the availability of working memory resources. Often the distinction between top-down and bottom-up effects of emotion is made in the context of the biased competition model of attentional selection (Desimone \& Duncan, 1995). According to the biased competition model stimuli compete for neural representation and this competitive process can be biased by either top-down goals or in a bottomup manner by the perceptual salience of the stimuli within the visual field. Top-down goals are typically used to refer to the task the participant is required to perform in the experiment (e.g., judge either the sex of a face or the emotion of a face). Based on the Biased Competition Model researchers have argued that emotional stimuli influence the attentional selection of neutral stimuli (Mather \& Sutherland, 2011) and are themselves prioritized for selection (Wang, Kennedy, \& Most, 2012). For an example of the former, the arousal-biased competition (ABC) model (Mather \& Sutherland, 2011) explains enhanced recall of perpetually salient (neutral) information by claiming that emotional arousal enhances the selection of goal-relevant and perceptually salient stimuli and concurrently suppresses the selection of competing, lower-priority stimuli (Sutherland \& Mather, 2012). The ABC model and other approaches do not consider speed-accuracy trade-off as possible top-down influence and therefore, it remains to be established whether such high-level decision making influences are needed to provide a complete description of the data that supports the ABC and other models (Bocanegra, 2014; Pessoa, 2015; Wang et al., 2012).

The current research raises the possibility that differences in decision making caution may have been present in previous studies of the effects of fearful and other expressions on perception. Despite this, I think it is unlikely that all previous effects are due to caution. This is because researchers have done more than simply recording RTs and accuracy following facial expressions, they have manipulated temporal attention (e.g., de Jong et al., 2010; Sigurjónsdóttir et al., 2015; Stebbins \& Vanous, 2015), spatial attention (e.g., Berggren et al., 2013; Bocanegra et al., 2012; Fox et al., 2002), perceptual load (Gupta et al., 2016; Hodsoll et al., 2011; Pessoa, Padmala, \& Morland, 2005) and other variables that are thought to affect the availability of attentional resources. The results of these studies are consistent with the prioritization of emotion stimuli for attentional selection and therefore, it would be remarkable if further analyses or research were to show that increased caution is responsible for these effects. Indeed, it is possible is that the effects of facial expressions on decision making caution and perceptual or attentional processes might co-exist. This is a possibility because the Diffusion Model indexes caution (or conservativeness) as a separate process from evidence accumulation and stimulus encoding. If the effects of facial expressions are mediated by attention then such effects will propagate onto the model parameters thought to affected by attention (Mulder \& van Maanen, 2013; Nunez, Vandekerckhove, \& Srinivasan, 2017; Smith \& Ratcliff, 2009). For example, research (see Figure 2; Mulder \& van Maanen, 2013). has shown that visual attention cues placed in the same versus an opposite location to a target both reduce non-decision times (supporting enhanced stimulus encoding) and increase the drift rate (supporting enhanced evidence accumulation). Therefore, in future studies it will be interesting to establish whether the effects of emotion on spatial attention will correspond to differences in the drift rate and non-decisions times. 
One unexpected result was the finding that non-decision times were longer and response caution was lower following sad compared to neutral expressions. Why might this pattern have emerged? One rating study (Horstmann \& Gernot, 2003) found that although facial expressions are typically described as expressing the emotional state of the sender - for example, fear expression are rated expressing a state of fear - they are sometimes perceived as behavioral intentions ("I am going to run away") or action requests (e.g., "help me"). For example sad expression were interpreted as a request for help or comfort and also as reflecting an intention to cry. Given such findings, one possibility is that people perceived sad expressions as a request for help and consequently allocated greater attention to sad compared to neutral expressions. Allocating attention to sad expression might have led to a processing bottleneck that delayed the encoding of the target (leading to longer non-decision times) and perhaps a subsequent hurried response in which participants traded accuracy for speed in an attempt to compensate for the delay. In other work (Tipples, in prep) I have recorded the longer no-decision times and reduced decision making caution for highly arousing emotion images - images that are likely to receive increased attention - and therefore, the pattern for sad expressions fits with this explanation.

There was no effect of sad expressions on the standard measures of RT and accuracy (although for the larger sample there was a small effect on $d^{\prime}$ ) and therefore, this research adds to the list of studies that have found that Diffusion Model can help identify process that are hard to detect using standard statistical methods (Krypotos, Beckers, Kindt, \& Wagenmakers, 2015; Pe, Vandekerckhove, \& Kuppens, 2013; White, Ratcliff, Vasey, \& McKoon, 2010). For example, one study (White et al., 2010) recorded a consistent processing advantage for threatening words in high-anxious individuals despite the fact such effects were absent when the data were analysed using traditional analyses of RTs and accuracy. Another study (Pe et al., 2013) found that rumination and depression scores were associated with facilitated processing due to negative distracters but failed to find any effect using either accuracy or RTs as separate measures. Combined with the current results finding these studies demonstrate the usefulness of the Diffusion Modelling to study emotion and perhaps emotion disorders (White, Skokin, Carlos, \& Weaver, 2016).

\section{Conclusion}

The research findings show that people make strategic adjustments in the evidence required to make a decision after see fearful and sad expressions - they are more cautious after seeing fear and less cautious after seeing sadness. For fearful expressions, the results concur with the idea (Whalen, 1998) that fearful expression warn of potential threat. Overall, the current results point a new direction for research in this area - testing the caution hypothesis using the Diffusion. 


\section{References}

Becker, M. W. (2009). Panic Search: Fear Produces Efficient Visual Search for Nonthreatening Objects. Psychological Science, 2O(4), 435-437. https://doi.org/10.1111/j.14679280.2009.02303.x

Berggren, N., Richards, A., Taylor, J., \& Derakshan, N. (2013). Affective attention under cognitive load: reduced emotional biases but emergent anxiety-related costs to inhibitory control. Frontiers in Human Neuroscience, 7. https://doi.org/10.3389/fnhum.2013.00188

Bocanegra, B. R. (2014). Affecting speed and accuracy in perception. Cognitive Affective \& Behavioral Neuroscience, 14(4), 1454-1466. https://doi.org/10.3758/s13415-014-0296-5

Bocanegra, B. R., Huijding, J., \& Zeelenberg, R. (2012). Beyond Attentional Bias: A Perceptual Bias in a Dot-Probe Task. Emotion, 12(6), 1362-1366. https://doi.org/10.1037/a0028415

Bocanegra, B. R., \& Zeelenberg, R. (2009a). Emotion Improves and Impairs Early Vision. Psychological Science, 20(6), 707-713. https://doi.org/10.1111/j.1467-9280.2009.02354.x

Bocanegra, B. R., \& Zeelenberg, R. (2009b). Emotion Improves and Impairs Early Vision.

Psychological Science, 2O(6), 707-713. https://doi.org/10.1111/j.1467-9280.2009.02354.x

Bocanegra, B. R., \& Zeelenberg, R. (2011). Emotion-Induced Trade-Offs in Spatiotemporal Vision. Journal of Experimental Psychology-General, 140(2), 272-282. https://doi.org/10.1037/a0023188

Borst, G., \& Kosslyn, S. M. (2010a). Fear selectively modulates visual mental imagery and visual perception. Quarterly Journal of Experimental Psychology, 63(5), 833-839. https://doi.org/10.1080/17470211003602420

Borst, G., \& Kosslyn, S. M. (2010b). Fear selectively modulates visual mental imagery and visual perception. Quarterly Journal of Experimental Psychology, 63(5), 833-839. https://doi.org/10.1080/17470211003602420

Bradley, B. P., Mogg, K., Falla, S. J., \& Hamilton, L. R. (1998). Attentional Bias for Threatening Facial Expressions in Anxiety: Manipulation of Stimulus Duration. Cognition \& Emotion, 12(6), 737-753. https://doi.org/10.1080/026999398379411

Breitmeyer, B. G., \& Williams, M. C. (1990). Effects of isoluminant-background color on metacontrast and stroboscopic motion: interactions between sustained (P) and transient (M) channels. Vision Research, 30(7), 1069-1075.

Callaway, E. M. (1998). Local circuits in primary visual cortex of the macaque monkey. Annual Review of Neuroscience, 21, 47-74. https://doi.org/10.1146/annurev.neuro.21.1.47

Carpenter, B., Gelman, A., Hoffman, M., Lee, D., Goodrich, B., Betancourt, M., ... Riddell, A. (in press). Stan: A probabilistic programming language.

Davis, F. C., Somerville, L. H., Ruberry, E. J., Berry, A. B. L., Shin, L. M., \& Whalen, P. J. (2011). A tale of two negatives: differential memory modulation by threat-related facial expressions. Emotion (Washington, D.C.), 11(3), 647-55. https://doi.org/10.1037/ao021625

de Jong, P. J., Koster, E. H. W., van Wees, R., \& Martens, S. (2010). Angry facial expressions hamper subsequent target identification. Emotion (Washington, D.C.), 1O(5), 727-732. https://doi.org/10.1037/aoo19353

Desimone, R., \& Duncan, J. (1995). Neural mechanisms of selective visual attention. Annual Review of Neuroscience, 18, 193-222.

https://doi.org/10.1146/annurev.ne.18.030195.001205

DIC. (n.d.). Retrieved 3 March 2017, from https://www.mrc-bsu.cam.ac.uk/software/bugs/thebugs-project-dic/

Dosher, B. A., \& Lu, Z.-L. (2000). Noise Exclusion in Spatial Attention. Psychological Science, 11(2), 139-146. https://doi.org/10.1111/1467-9280.00229

Ekman, P., \& Friesen, W. V. (1976). Pictures of facial affect. Palo Alto, Calif: Consulting Psychologists Press.

Fox, E., Russo, R., \& Dutton, K. (2002). Attentional Bias for Threat: Evidence for Delayed Disengagement from Emotional Faces. Cognition \& Emotion, 16(3), 355-379.

Gabry, J., University, T. of C., (R/pp_data.R, R. C. D. T., R/stan_aov.R), (R/pp_data.R), D. B., (R/pp_data.R), M. M., ... Goodrich, B. (2016). rstanarm: Bayesian Applied Regression 
Modeling via Stan (Version 2.10.1). Retrieved from https://cran.rproject.org/web/packages/rstanarm/index.html

Gelman, A., Carlin, J., Stern, H., Dunson, D., Vehtari, A., \& Rubin, D. (2014). Bayesian Data Analysis, Third Edition (Chapman \& $\{\mathrm{Hall} / \mathrm{CRC}\}$ Texts in Statistical Science). Chapman and Hall/CRC.

Georgiou, G. A., Bleakley, C., Hayward, J., Russo, R., Dutton, K., Eltiti, S., \& Fox, E. (2005). Focusing on fear: Attentional disengagement from emotional faces. Visual Cognition, 12(1), 145-158. https://doi.org/10.1080/13506280444000076

Gupta, R., Hur, Y.-J., \& Lavie, N. (2016). Distracted by pleasure: Effects of positive versus negative valence on emotional capture under load. Emotion (Washington, D.C.), 16(3), 328-337. https://doi.org/10.1037/emoooo0112

Hodsoll, S., Viding, E., \& Lavie, N. (2011). Attentional capture by irrelevant emotional distractor faces. Emotion (Washington, D.C.), 11(2), 346-353. https://doi.org/10.1037/a0022771

Horstmann, G., \& Gernot. (2003). What do facial expressions convey: Feeling states, behavioral intentions, or actions requests? Emotion, 3(2), 150-166. https://doi.org/10.1037/15283542.3.2.150

Krypotos, A.-M., Beckers, T., Kindt, M., \& Wagenmakers, E.-J. (2015). A Bayesian hierarchical diffusion model decomposition of performance in Approach-Avoidance Tasks. Cognition \& Emotion, 29(8), 1424-1444. https://doi.org/10.1080/02699931.2014.985635

Lee, T.-H., Baek, J., Lu, Z.-L., \& Mather, M. (2014a). How Arousal Modulates the Visual Contrast Sensitivity Function. Emotion, 14(5), 978-984. https://doi.org/10.1037/a0037047

Lee, T.-H., Baek, J., Lu, Z.-L., \& Mather, M. (2014b). How arousal modulates the visual contrast sensitivity function. Emotion, 14(5), 978-984. https://doi.org/10.1037/ao037047

Lojowska, M., Gladwin, T. E., Hermans, E. J., \& Roelofs, K. (2015). Freezing Promotes Perception of Coarse Visual Features. Journal of Experimental Psychology-General, 144(6), 10801088. https://doi.org/10.1037/xgeoooo117

Macmillan, N. A., \& Creelman, C. D. (2005). Detection theory: a user's guide. Mahwah, NY. ; London: Lawrence Erlbaum Associates.

Maratos, F. A. (2011). Temporal processing of emotional stimuli: The capture and release of attention by angry faces. Emotion, 11(5), 1242-1247. https://doi.org/10.1037/a0024279

Mather, M., \& Sutherland, M. R. (2011). Arousal-Biased Competition in Perception and Memory. Perspectives on Psychological Science: A Journal of the Association for Psychological Science, 6(2), 114-133. https://doi.org/10.1177/1745691611400234

Matzke, D., \& Wagenmakers, E.-J. (2009). Psychological interpretation of the ex-Gaussian and shifted Wald parameters: A diffusion model analysis. Psychonomic Bulletin \& Review, 16(5), 798-817. https://doi.org/10.3758/PBR.16.5.798

Mogg, K., \& Bradley, B. P. (2016). Anxiety and attention to threat: Cognitive mechanisms and treatment with attention bias modification. Behaviour Research and Therapy, 87, 76-108. https://doi.org/10.1016/j.brat.2016.08.001

Mulder, M. J., \& van Maanen, L. (2013). Are Accuracy and Reaction Time Affected via Different Processes? PLoS ONE, 8(11), e80222. https://doi.org/10.1371/journal.pone.0080222

Nicol, J. R., Perrotta, S., Caliciuri, S., \& Wachowiak, M. P. (2013a). Emotion-specific modulation of early visual perception. Cognition \& Emotion, 27(8), 1478-1485.

Nicol, J. R., Perrotta, S., Caliciuri, S., \& Wachowiak, M. P. (2013b). Emotion-specific modulation of early visual perception. Cognition \& Emotion, 27(8), 1478-1485.

Nosek, B. A., Spies, J. R., \& Motyl, M. (2012). Scientific Utopia: II. Restructuring incentives and practices to promote truth over publishability. arXiv:1205.4251 [Physics]. Retrieved from http://arxiv.org/abs/1205.4251

Nunez, M. D., Vandekerckhove, J., \& Srinivasan, R. (2017). How attention influences perceptual decision making: Single-trial EEG correlates of drift-diffusion model parameters. Journal of Mathematical Psychology, 76, 117-130. https://doi.org/10.1016/j.jmp.2016.03.003

Pe, M. L., Vandekerckhove, J., \& Kuppens, P. (2013). A Diffusion Model Account of the Relationship Between the Emotional Flanker Task and Rumination and Depression. Emotion, 13(4), 739-747. https://doi.org/10.1037/a0031628 
Peers, P. V., \& Lawrence, A. D. (2009). Attentional control of emotional distraction in rapid serial visual presentation. Emotion, 9(1), 140-145. https://doi.org/10.1037/a0014507

Peirce, J. W. (2007). PsychoPy-Psychophysics software in Python. Journal of Neuroscience Methods, 162(1-2), 8-13. https://doi.org/10.1016/j.jneumeth.2006.11.017

Pessoa, L. (2015). Précis on The Cognitive-Emotional Brain. The Behavioral and Brain Sciences, 38, e71. https://doi.org/10.1017/So140525X14000120

Pessoa, L., Padmala, S., \& Morland, T. (2005). Fate of unattended fearful faces in the amygdala is determined by both attentional resources and cognitive modulation. NeuroImage, 28(1), 249-255. https://doi.org/10.1016/j.neuroimage.2005.05.048

Quinlan, P. T., \& Johnson, D. P. (2011). The effect of inducing panic search on the detection of fear-relevant and neutral images. Visual Cognition, 19(6), 762-784. https://doi.org/10.1080/13506285.2011.587846

Ratcliff, R. (1978). A theory of memory retrieval. Psychological Review, 85(2), 59-108. https://doi.org/10.1037/0033-295X.85.2.59

Ratcliff, R., \& McKoon, G. (2008). The diffusion decision model: theory and data for two-choice decision tasks. Neural Computation, 2O(4), 873-922.

https://doi.org/10.1162/neco.2008.12-06-420

Schouten, J. F., \& Bekker, J. A. (1967). Reaction time and accuracy. Acta Psychologica, 27, 143153.

Sigurjónsdóttir, Ó., Sigurðardóttir, S., Björnsson, A. S., \& Kristjánsson, Á. (2015). Barking up the wrong tree in attentional bias modification? Comparing the sensitivity of four tasks to attentional biases. Journal of Behavior Therapy and Experimental Psychiatry, 48, 9-16. https://doi.org/10.1016/j.jbtep.2015.01.005

Simon, H. (1971). Designing Organizations for an Information-Rich World. In M. Greenberger (Ed.), Computers, communications, and the public interest (pp. 37-72).

Smith, P. L., \& Ratcliff, R. (2009). An integrated theory of attention and decision making in visual signal detection. Psychological Review, 116(2), 283-317. https://doi.org/10.1037/a0015156

Song, I., \& Keil, A. (2013). Affective Engagement and Subsequent Visual Processing: Effects of Contrast and Spatial Frequency. Emotion, 13(4), 748-757.

https://doi.org/10.1037/aoo31553

Spiegelhalter, D. J., Best, N. G., Carlin, B. P., \& Van Der Linde, A. (2002). Bayesian measures of model complexity and fit. Journal of the Royal Statistical Society: Series B (Statistical Methodology), 64(4), 583-639. https://doi.org/10.1111/1467-9868.00353

Stanislaw, H., \& Todorov, N. (1999). Calculation of signal detection theory measures. Behavior Research Methods, Instruments, \& Computers, 31(1), 137-149. https://doi.org/10.3758/BFo3207704

Stebbins, H. E., \& Vanous, J. B. (2015). The influence of stimulus sex and emotional expression on the attentional blink. Emotion (Washington, D.C.), 15(4), 511-521. https://doi.org/10.1037/emoooooo82

Stoet, G. (2010). PsyToolkit: A software package for programming psychological experiments using Linux. Behavior Research Methods, 42(4), 1096-1104. https://doi.org/10.3758/BRM.42.4.1096

Sutherland, M. R., \& Mather, M. (2012). Negative arousal amplifies the effects of saliency in shortterm memory. Emotion, 12(6), 1367-1372. https://doi.org/10.1037/a0027860

Taylor, J. M., \& Whalen, P. J. (2014). Fearful, but not angry, expressions diffuse attention to peripheral targets in an attentional blink paradigm. Emotion (Washington, D.C.), 14(3), 462-8. https://doi.org/10.1037/aoo36034

Van Dillen, L. F., \& Koole, S. L. (2009). How automatic is 'automatic vigilance'? The role of working memory in attentional interference of negative information. Cognition and Emotion, 23(6), 1106-1117. https://doi.org/10.1080/02699930802338178

Vandekerckhove, J., Tuerlinckx, F., \& Lee, M. (2008, July). A Bayesian approach to diffusion process models of decision-making. 
Vandekerckhove, J., Tuerlinckx, F., \& Lee, M. D. (2011a). Hierarchical diffusion models for twochoice response times. Psychological Methods, 16(1), 44-62. https://doi.org/10.1037/a0021765

Vandekerckhove, J., Tuerlinckx, F., \& Lee, M. D. (2011b). Hierarchical diffusion models for twochoice response times. Psychological Methods, 16(1), 44-62. https://doi.org/10.1037/a0021765

Vermeulen, N., Godefroid, J., \& Mermillod, M. (2009). Emotional Modulation of Attention: Fear Increases but Disgust Reduces the Attentional Blink. PLoS ONE, 4(11), e7924. https://doi.org/10.1371/journal.pone.0007924

Voss, A., Voss, J., \& Lerche, V. (2015). Assessing cognitive processes with diffusion model analyses: a tutorial based on fast-dm-30. Frontiers in Psychology, 6. https://doi.org/10.3389/fpsyg.2015.00336

Wang, L., Kennedy, B. L., \& Most, S. B. (2012). When Emotion Blinds: A Spatiotemporal Competition Account of Emotion-Induced Blindness. Frontiers in Psychology, 3. https://doi.org/10.3389/fpsyg.2012.00438

Whalen, P. J. (1998). Fear, Vigilance, and Ambiguity: Initial Neuroimaging Studies of the Human Amygdala. Current Directions in Psychological Science, 7(6), 177-188. https://doi.org/10.1111/1467-8721.ep10836912

White, C. N., Ratcliff, R., Vasey, M. W., \& McKoon, G. (2010). Anxiety enhances threat processing without competition among multiple inputs: A diffusion model analysis. Emotion, 1O(5), 662-677. https://doi.org/10.1037/ao019474

White, C. N., Skokin, K., Carlos, B., \& Weaver, A. (2016). Using Decision Models to Decompose Anxiety-Related Bias in Threat Classification. Emotion, 16(2), 196-203. https://doi.org/10.1037/emooooo109

Wickelgren, W. A. (1977). Speed-accuracy tradeoff and information processing dynamics. Acta Psychologica, 41(1), 67-85. https://doi.org/10.1016/0001-6918(77)90012-9

Wiecki, T. V, Sofer, I., \& Frank, M. J. (2013). HDDM: Hierarchical Bayesian estimation of the Drift-Diffusion Model in Python. Frontiers in Neuroinformatics, 7, 14. https://doi.org/10.3389/fninf.2013.00014

Yiend, J. (2010). The effects of emotion on attention: A review of attentional processing of emotional information. Cognition and Emotion, 24(1), 3-47. https://doi.org/10.1080/02699930903205698

Zeelenberg, R., \& Bocanegra, B. R. (2010). Auditory emotional cues enhance visual perception. Cognition, 115(1), 202-206. https://doi.org/10.1016/j.cognition.2009.12.004 


\section{Figure Captions}

Figure 1. The Diffusion Model (Ratcliff, 1978; Ratcliff \& McKoon, 2008). Evidence accumulation begins at a starting point $\mathrm{z}$, and proceeds toward absorbing boundaries that determine when the decision criterion is met and the accumulation process ends. The drift rate indicates how fast information accumulates toward a boundary. Non-decision times are modelled as the additional time needed for processing before and after the accumulation process. Non-decision time includes the time to encode the stimulus and prepare a response. Further parameters can also be included in the model (e.g., trial-by-trial variability in the starting point parameter z).

Figure 2-Posterior distributions for the boundary separation parameter for the fearful and neutral face conditions for Experiment 1 (2a) and Experiment 2 (2b).

Figure 3 - Mean estimated effect of expression on boundary separation values for Experiments 1 (fearful vs neutral), 2 (fearful vs neutral) and 4 (sadness vs neutral). Positive values indicate increased caution. Error bars are 95\% Credibility intervals for the estimated effect. A difference of zero is represented as a dashed horizontal line at zero on the y axis.

Figure 4-Posterior distributions for the boundary separation parameter (4a) and non-decision times (4b) for the sad and neutral face conditions for Experiment 4. 